\title{
Parity inversion and breakdown of shell closure in Be isotopes
}

\author{
G. Gori, ${ }^{1,2}$ F. Barranco, ${ }^{3}$ E. Vigezzi, ${ }^{2}$ and R. A. Broglia ${ }^{1,2,4}$ \\ ${ }^{1}$ Dipartimento di Fisica, Università degli Studi di Milano, via Celoria 16, 20133 Milano, Italy \\ ${ }^{2}$ INFN, Sezione di Milano, via Celoria 16, 20133 Milano, Italy \\ ${ }^{3}$ Departamento de Fisica Aplicada III, Escuela Superior de Ingenieros, Camino de los Descubrimientos s/n, 41092 Sevilla, Spain \\ ${ }^{4}$ The Niels Bohr Institute, University of Copenhagen, Blegdamsvej 17, 2100 Copenhagen Ø, Denmark \\ (Received 18 April 2003; revised manuscript received 17 October 2003; published 19 April 2004)
}

\begin{abstract}
The coupling of single-particle motion and of vibrations in ${ }_{4}^{11} \mathrm{Be}$ produces dressed neutrons which spend only a fraction of the time in pure single-particle states, and which weighing differently from the bare neutrons lead to parity inversion. The interaction of the two least bound neutrons in the ground state of ${ }_{4}^{12} \mathrm{Be}$ mediated by the $v_{14}$ Argonne nucleon-nucleon potential and by the exchange of surface vibrations of the core ${ }^{10} \mathrm{Be}$ gives rise to a strongly correlated state, where the two valence neutrons are distributed over $s^{2}, p^{2}$, and $d^{2}$ configurations, resulting in the breaking of the $N=8$ shell closure.
\end{abstract}

DOI: 10.1103/PhysRevC.69.041302

PACS number(s): 21.60.Ev, 21.10.-k, 21.60.Jz, 27.20.+n

If neutrons are progressively added to a normal nucleus, the Pauli principle forces them into states of higher momentum. When the core becomes neutron saturated the nucleus expels most of the wave function of the last neutrons outside to form a halo, which because of its large size can have lower momentum. It is an open question how nature stabilizes such a fragile system and provides the glue needed to bind the halo neutrons to the core. Here we show that this problem is similar to that of the instability of the normal state of an electron system at zero temperature solved by Cooper, solution which is on the basis of BCS theory of superconductivity. To understand the origin and the consequences of pairing correlations, it is illustrative to study the problem of two electrons interacting on top of a noninteracting Fermi sea of electrons. Thus, all but two of the electrons are assumed to be noninteracting. The background of electrons enter the problem only through the Pauli principle by blocking states below the Fermi surface from participating in the twoelectron problem.

This system, first studied by Cooper [1], is unstable against the formation of a bound electron pair, regardless of how weak the interaction is, so long as it is attractive. This result is a consequence of the Fermi statistics and of the existence of the Fermi-sea background, since it is well known that binding does not ordinarily occur in the twobody problem in three dimensions until the strength of the potential exceeds a finite threshold value.

Although actual superconductors differ in a fundamental way from a single bound pair model, Cooper pairs can be viewed as the building blocks of the superconductor. Furthermore, in the nuclear case, where the number of fermions participating in the condensate is small, and where the pairing problem can be studied in terms of individual quantal states, the Cooper pair problem can describe realistic situations. In fact it seems to have a concrete realization in the halo nucleus ${ }^{12} \mathrm{Be}\left({ }^{10} \mathrm{Be}+2 n\right)$, which can be viewed as two weakly bound neutrons moving around the core ${ }^{10} \mathrm{Be}$. By allowing these two neutrons to interact through the bare nucleon-nucleon potential, and to exchange surface vibra- tions of the core, one is able to provide a unified and quantitative picture of the observed properties of this system. This result, which is quite general, suggests a strategy for designing nuclei at the edges of the neutron drip line, and thus probing the limits of nuclear stability. It also provides evidence of the limits of validity of BCS theory in finite systems: a single Cooper pair.

The properties of finite many-body systems are strongly influenced by spatial quantization [2] leading to marked shell structures [3]. This type of quantal size effects are, on the other hand, renormalized in an important way by zero point fluctuations [4,5]. The smaller the system is, the larger the surface/volume ratio is and the stronger these effects may become. In particular, the interweaving of single-particle motion and of collective vibrations of the surface of atomic nuclei can lead to state-dependent effective masses which for light systems can even invert the sequence of particular levels as compared with the predictions of mean-field theory, thus altering the magic numbers associated with closed shells. While the standard parametrization of the singleparticle potential [3] indicates the states $0 p_{1 / 2}$ and $1 s_{1 / 2}$ to be the last bound and the first unbound orbitals of the system with $Z=4$ and $N=7$ nucleons, ${ }_{4}^{11} \mathrm{Be}_{7}$ displays parity inversion with respect to this sequence of single-particle levels. In fact, the two bound states of this system have quantum numbers (binding energy) $1 / 2^{+}(-0.5 \mathrm{MeV})$ and $1 / 2^{-}(-0.18 \mathrm{MeV})$, respectively [6]. The associated spectroscopic factors measured in the knockout reaction ${ }_{4}^{12} \mathrm{Be}_{8}\left({ }^{9} \mathrm{Be},{ }^{9} \mathrm{Be}+n+\gamma\right){ }^{11} \mathrm{Be}$ are $0.42 \pm 0.1$ and $0.37 \pm 0.1$, respectively [7], indicating that $N=8$ is not a good magic number in the case of the neutron rich nucleus ${ }^{12} \mathrm{Be}$, as it is not in the case of ${ }^{11} \mathrm{Li}$ either.

Making use of the fact that the effective mass ( $\omega$ mass) $m_{\omega}=m(1+\lambda)[8]$, arising from the coupling of single-particle states to collective vibrations of the system as measured by the mass enhancement factor $\lambda$, is closely connected with the associated spectroscopic factor $Z_{\omega}=m / m_{\omega}=(1+\lambda)^{-1}$ of levels close to the Fermi energy, and of the experimental finding quoted above $\left(Z_{\omega} \approx 0.4-0.5\right)$ one estimates $\lambda \approx 1.2$. Adopting this value, the two-particle separation energy associated 
to the least bound neutrons of ${ }^{12} \mathrm{Be}$ is expected to be $W$ $\approx-\lambda \omega_{C}$ (strong coupling limit of the Cooper pair solution [9]), where $\omega_{C}$ is a typical energy of vibrational modes $\left(\approx 3.37 \mathrm{MeV}\right.$, quadrupole vibration in $\left.{ }^{10} \mathrm{Be}[10]\right)$. Consequently $W \approx-4 \mathrm{MeV}$, a value which agrees pretty well with the experimental finding $\left(S_{2 n}=-3.7 \mathrm{MeV}\right.$ [11]). From these simple estimates, one expects the renormalization effects of the nucleon-nucleon interaction (induced interaction) of the two least bound neutrons moving around the core ${ }^{10} \mathrm{Be}$ due to the particle-vibration phenomenon, to be conspicuous.

In what follows we shall study the nuclei ${ }_{4}^{11} \mathrm{Be}_{7}$ and ${ }_{4}^{12} \mathrm{Be}_{8}$, within the framework of nuclear field theory (NFT) (cf. Ref. [12], and references therein), taking thus into account the coupling between single-particle motion and collective vibrations of the system. In the case of ${ }^{12} \mathrm{Be}$ we also include the direct interaction between the two halo nucleons through a nucleon-nucleon realistic potential (Argonne $v_{14}$ [13]). Special emphasis will be set in the calculation of the spectroscopic factors of ${ }^{12} \mathrm{Be}$ which, together with the ground state occupation probabilities of the two-particle configurations $s^{2}, p^{2}$, and $d^{2}$, provide the most sensitive quantities for a detailed comparison with the experimental findings.

We start by considering the system ${ }_{4}^{11} \mathrm{Be}_{7}$ described as one neutron moving around the core ${ }_{4}^{10} \mathrm{Be}_{6}$, in keeping with the fact that the value of the neutron separation energy in ${ }^{10} \mathrm{Be}$ is $6.813 \mathrm{MeV}$ as compared with the value of $0.504 \mathrm{MeV}$ in ${ }^{11} \mathrm{Be}$ [11]. The description of the system is carried out in a space given by the tensor product of single-particle states $a_{\nu}^{\dagger}|-\rangle_{F}$ and collective modes $\Gamma_{\lambda \mu}^{\dagger}|-\rangle_{B}$. The associated Hamiltonian is

$$
\hat{H}_{p v}=\hat{H}_{F}+\hat{H}_{B}+\delta \hat{U}
$$

where

$$
\hat{H}_{F}=\sum_{\nu} \epsilon_{\nu} a_{\nu}^{\dagger} a_{\nu}
$$

is the mean field acting on the fermionic degrees of freedom. The single-particle energies $\epsilon_{\nu}$ are determined by solving the Schrödinger equation

$$
\left(-\frac{\hbar^{2}}{2 m_{k}} \nabla_{r}^{2}+\frac{m}{m_{k}} U(r)\right) \phi_{\nu}(r)=\epsilon_{\nu} \phi_{\nu}(r)
$$

in a spherical box of radius $R_{b o x}$. The potential $U(r)$ is parametrized according to Ref. [3], and is the sum of a central potential of Saxon-Woods shape with a depth given by $V$ $=-50.5+33(N-Z) / A \mathrm{MeV}$ and of a spin-orbit term. This potential gives a good average reproduction of the singleparticle levels along the valley of stability, using an effective mass equal to the bare mass, $m_{k}=m$ in Eq. (3). In the present calculation, one could argue that as we include explicitly the renormalization due to the coupling of the phonon, we should use for $m_{k}$ the value associated to a

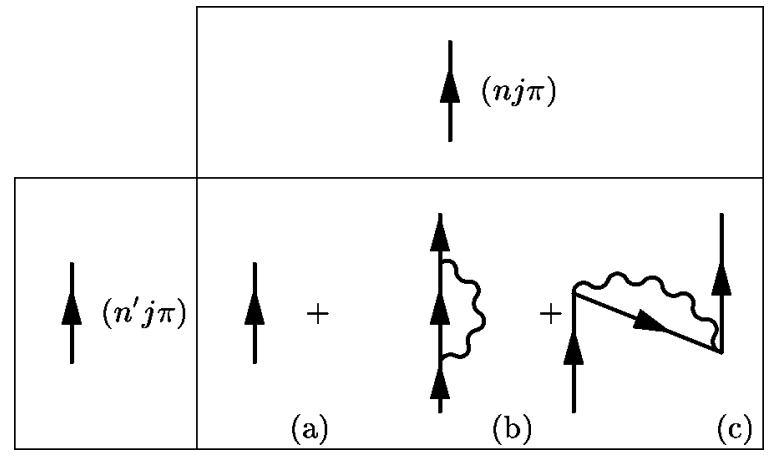

FIG. 1. Schematic representation of the effective matrix used in the Bloch-Horowitz perturbation theory to calculate the eigenvalues of ${ }^{11} \mathrm{Be}$. An arrowed line pointing upwards (downwards) indicate a particle (hole), while a wavy line indicate a collective vibrational state.

Hartree-Fock calculation. For well-bound nuclei, the value of $m_{k}$ would be reduced to $m_{k} \approx 0.7 m$ due the effect of the exchange (Fock) term. In the present case, however, the halo neutrons lie significantly outside the central core and we expect a higher value of $m_{k}$. Anyhow, we have found that our main results are not sensitive to the precise value of $m_{k}$ in the interval between 0.8 and 1 , and in the calculations reported in the following we have used $m_{k}=m$. In our calculation we have included the hole $p_{3 / 2}$ orbital occupied in the ${ }^{10} \mathrm{Be}$ core, and the $s_{1 / 2}, p_{1 / 2}$, and $d_{5 / 2}$ unoccupied orbitals, with energies up to $E_{\max }=350 \mathrm{MeV}$, in a box of radius $R_{b o x}=30 \mathrm{fm}$.

The term $\hat{H}_{B}$ in Eq. (1) is the Hamiltonian associated to the dynamics of the core. We shall adopt a harmonic approximation, calculating the excited states of ${ }^{10} \mathrm{Be}$ by means of the quasiparticle random phase approximation (QRPA) [14] using a separable multipole-multipole interaction with a coupling constant determined by means of the self-consistent condition of Bohr and Mottelson [4]. We have slightly adjusted its value in order to obtain the transition strength of the low-lying $2^{+}$state in better agreement with the experimental values [10].

The third term in Eq. (1), $\delta \hat{U}$, is the term coupling the single-particle motion with the surface vibrations:

$$
\begin{aligned}
\delta \hat{U}= & \sum_{\lambda \mu} \sqrt{\frac{\hbar}{2 \omega_{\lambda} B_{\lambda}}(i)^{-\lambda}\left\{\left[\hat{\Gamma}_{\lambda \mu}^{\dagger}+(-)^{\lambda+\mu} \hat{\Gamma}_{\lambda-\mu}\right]\right.} \\
& \left.\times \sum_{k k^{\prime}}\left\langle k\left|-R_{0} \frac{\partial U}{\partial r} Y_{\lambda \mu}^{*}\right| k^{\prime}\right\rangle a_{k}^{\dagger} a_{k^{\prime}}\right\} .
\end{aligned}
$$

The operators $\hat{\Gamma}_{\lambda \mu}^{\dagger}$ and $\hat{\Gamma}_{\lambda \mu}$, respectively, create and destroy a quantum of vibration in the core, having angular momentum and projection $\lambda, \mu$. The phonon energies $\hbar \omega_{\lambda}$ and transition strengths $\sqrt{\hbar / 2 B_{\lambda} \omega_{\lambda}}=\beta_{\lambda} / \sqrt{2 \lambda+1}$ are obtained from the QRPA calculation. In the calculations for ${ }^{11} \mathrm{Be}$, we have 
TABLE I. Comparison of experimental binding energy and spectroscopic factors with those resulting from our calculation and from an independent particle model. For ${ }^{12} \mathrm{Be}$, we also show the components of the resulting ground state wave function. The experimental spin-parity assignment of the state at $1.28 \mathrm{MeV}$ in ${ }^{11} \mathrm{Be}$ is only tentative $[18,21]$.

\begin{tabular}{|c|c|c|c|c|}
\hline & & \multirow[b]{2}{*}{ Expt. } & \multicolumn{2}{|c|}{ Theory } \\
\hline & & & Particle vibration & Mean field \\
\hline \multirow{9}{*}{${ }_{4}^{11} \mathrm{Be}_{7}$} & $E_{s_{1 / 2}}$ & $-0.504 \mathrm{MeV}$ & $-0.48 \mathrm{MeV}$ & $\sim 0.14 \mathrm{MeV}$ \\
\hline & $E_{p_{1 / 2}}$ & $-0.18 \mathrm{MeV}$ & $-0.27 \mathrm{MeV}$ & $-3.12 \mathrm{MeV}$ \\
\hline & $E_{d_{5 / 2}}$ & $1.28 \mathrm{MeV}$ & $\sim 0 \mathrm{MeV}$ & $\sim 2.4 \mathrm{MeV}$ \\
\hline & $S\left[1 / 2^{+}\right]$ & $0.65-0.80[19]$ & 0.87 & 1 \\
\hline & & $0.73 \pm 0.06[20]$ & & \\
\hline & & $0.77[21]$ & & \\
\hline & $S\left[1 / 2^{-}\right]$ & $0.63 \pm 0.15[20]$ & 0.96 & 1 \\
\hline & & $0.96[21]$ & & 1 \\
\hline & $S\left[5 / 2^{+}\right]$ & & 0.72 & 1 \\
\hline \multirow{4}{*}{${ }_{4}^{12} \mathrm{Be}_{8}$} & $S_{2 n}$ & $-3.673 \mathrm{MeV}$ & $-3.58 \mathrm{MeV}$ & $-6.24 \mathrm{MeV}$ \\
\hline & $s^{2}, p^{2}, d^{2}$ & & $23 \%, 29 \%, 48 \%$ & $0 \%, 100 \%, 0 \%$ \\
\hline & $S\left[1 / 2^{+}\right]$ & $0.42 \pm 0.10[7]$ & 0.31 & 0 \\
\hline & $S\left[1 / 2^{-}\right]$ & $0.37 \pm 0.10[7]$ & 0.57 & 2 \\
\hline
\end{tabular}

included the coupling to the $2^{+}$and $3^{-}$phonons; neglecting the $3^{-}$states only slightly affects the results.

The interweaving of single-particle motion and of collective degrees of freedom arising from the particle-vibration Hamiltonian has been treated within the framework of the NFT making use of Bloch-Horowitz perturbation theory $[12,15]$. The eigenvalues of the dressed single-particle states were thus obtained by diagonalizing (energy dependent) matrices of the order of $10^{2} \times 10^{2}$ (cf. Fig. 1) whose elements connect a basis of unperturbed states containing both bound and continuum solutions of Eq. (3), corresponding to the quantum numbers $s_{1 / 2}, p_{1 / 2}$, and $d_{5 / 2}$.

The process depicted in Fig. 1(b) describes the emission and reabsorption of a phonon by a valence neutron having angular momentum and parity $j \pi$, and leads to an increase of the binding energy of the neutron. The process of Fig. 1(c) accounts instead for the indistinguishibility of the ${ }^{10} \mathrm{Be}$ core and of the valence neutrons, that is, for the Pauli principle. Due to the particle-vibration coupling, there will be processes contributing to the ground state energy of ${ }^{10} \mathrm{Be}$, in which a neutron, initially in a hole state below the Fermi energy, virtually emits a phonon and jumps into an unoccupied state $j \pi$ above the Fermi energy, finally returning to its initial state by reabsorbing the phonon. This process becomes forbidden when a valence neutron is added in the state $j \pi$ to form ${ }^{11} \mathrm{Be}$. Therefore the correlation energy associated to the core fluctuation must be subtracted in calculating the binding energy of the valence neutron in ${ }^{11} \mathrm{Be}$, and this is precisely the contribution of Fig. 1(c) [9].

The self-energy process (b) represented in Fig. 1 involves mainly the positive parity $1 / 2^{+}$and $5 / 2^{+}$states lying close to the Fermi energy, lowering their energy through the coupling to the $2^{+}$core excitation. On the other hand, the contributions associated to the graph (c) of Fig. 1, concern mostly the $1 / 2^{-}$ state, and lead to a reduction of its binding energy, because its partial occupation in ${ }^{11} \mathrm{Be}$ and in ${ }^{12} \mathrm{Be}$ blocks the ground state correlations of the ${ }^{10} \mathrm{Be}$ core. In fact, the blocking of transitions from the hole state $1 p_{3 / 2}$ to the particle state $1 p_{1 / 2}$ reduces the energy of the $1 / 2^{-}$state by about $3 \mathrm{MeV}$, and turns out to be essential to explain the parity inversion observed in ${ }^{11} \mathrm{Be}$. The final results for the single-particle energies are listed in Table I, in comparison with the experimental findings. The calculated matter radius is $3.2 \mathrm{fm}$, to be compared with the experimental value of $2.9 \pm 0.05$ [16]. The resulting wave function for the $1 / 2^{+}$ground state has a $15 \%$ admixture with the $\left(d_{5 / 2} \times 2^{+} ; 1 / 2^{+}\right)$configuration. This value compares well with the experimental information which has recently become available through the measurement of the $\left({ }^{11} \mathrm{Be},{ }^{10} \mathrm{Be}+\gamma\right)$ knockout reaction [17] and of the population of the $2^{+}$state of ${ }^{10} \mathrm{Be}$ in the $p\left({ }^{11} \mathrm{Be},{ }^{10} \mathrm{Be}\right) d$ one-neutron transfer reaction [19] (in the latter case a "best estimate" of $16 \%$ was obtained for the admixture). From our wave function we have also obtained the spectroscopic factors $S\left[1 / 2^{+}\right]$ and $S\left[1 / 2^{-}\right]$associated with the reaction ${ }^{10} \mathrm{Be}(d, p){ }^{11} \mathrm{Be}$ [20,21], populating both the $1 / 2^{+}$and $1 / 2^{-}$levels in ${ }^{11} \mathrm{Be}$; the calculation is discussed below in connection with a similar calculation carried out for the reaction ${ }^{12} \mathrm{Be}\left({ }^{9} \mathrm{Be},{ }^{9} \mathrm{Be}+n\right.$ $+\gamma){ }^{11} \mathrm{Be}$ [7]. The value of $S\left[1 / 2^{+}\right]$can also be inferred from the population of the $0^{+}$state in the $p\left({ }^{11} \mathrm{Be},{ }^{10} \mathrm{Be}\right) d$ oneneutron transfer reaction [19]. The extraction of the spectroscopic factors depends on the theoretical model assumed for the reaction analysis so that the experimental values listed in Table I are subject to a rather large uncertainty. In particular, the analysis of Ref. [22] leads, in the case of ${ }^{11} \mathrm{Be}$, to much smaller values $\left(S\left[1 / 2^{+}\right]=0.36-0.44\right)$. 


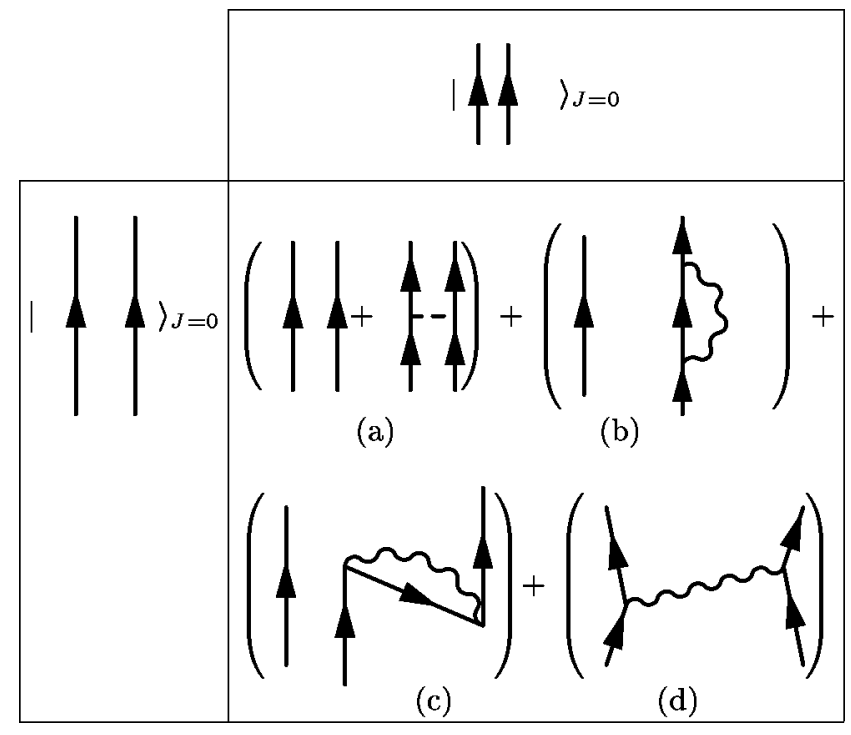

FIG. 2. Schematic representation of the effective matrix used in the Bloch-Horowitz perturbation theory to calculate the eigenvalues of ${ }^{12} \mathrm{Be}$. The dashed horizontal line represents the bare (Argonne $v_{14}$ ) nucleon-nucleon potential. Pairs of nucleons are coupled to angular momentum $J=0$.

The calculations reported above differ in some important respects from previous works which have also investigated the structure of ${ }^{11} \mathrm{Be}$, on the basis of particle-vibration coupling [24]. In particular, the effect of Pauli blocking, represented by graph (c), was not included in the calculations of Refs. [26-28], where instead an ad hoc, strong increase of the spin-orbit potential was introduced in order to reproduce the experimental energy of the $1 / 2^{-}$ state. We want also to stress the importance of including a sufficient number of excited continuum states in the calculation: their interference leads to a localized state, so that the result converges with the size of the box (contrary to the result of Ref. [26]). This applies, in particular, to the case of the $s_{1 / 2}$ state, which is unbound in our initial mean-field potential (cf. Table I), and is essential in the case of ${ }^{11} \mathrm{Li}$ [25].

The self-energy processes used to describe the dressed single-particle states of ${ }^{11} \mathrm{Be}$ have been included in the description of the ground state of ${ }^{12} \mathrm{Be}$. As in the case of ${ }^{11} \mathrm{Be}$, we use the Bloch-Horowitz and NFT rules to build the effective matrix to be diagonalized (of the order of $10^{3} \times 10^{3}$ ), which is shown in Fig. 2. The Hilbert space used to describe ${ }^{12} \mathrm{Be}$ is made out of two-particle states [cf. Fig. 2(a)], two particles and one phonon [Fig. 2(b) and 2(d)], and three particles-one hole and one phonon states [Fig. 2(c)], all coupled to zero angular momentum. In this case, however, we also add the bare nucleon-nucleon $v_{14}$ interaction, acting between the two halo neutrons in the ${ }^{1} S_{0}$ channel. Because of the repulsive core associated with the bare nucleon-nucleon ${ }^{1} S_{0}$ potential [cf. Fig. 2(a)], single-particle states with energies up to $500 \mathrm{MeV}$ were used in the calculations to ensure convergence of the results.
The two valence neutrons were coupled to the phonons of ${ }^{12} \mathrm{Be}$ itself, rather than to those of ${ }^{10} \mathrm{Be}$ core. In fact, the presence of a second valence neutron renormalizes in a strong way the properties of the phonons. This renormalization could be taken into account by NFT, but this would require a rather involved calculation. We have instead fixed the parameters of QRPA in order to reproduce the experimental properties of the low-lying vibrations of ${ }^{12} \mathrm{Be}$. Besides the $2^{+}$and the $3^{-}$phonons, also the $1^{-}$phonons were included to account for recoil terms [29] and the coupling constants needed to define the multipole-multipole residual interaction were tuned in order to reproduce the energies of the lowest $1^{-}$and $2^{+}$states measured in this nucleus $[10,30]$.

The lowest eigenvalue of the matrix depicted in Fig. 2 is $-3.58 \mathrm{MeV}$, to be compared with the experimental twoparticle separation energy of $-3.67 \mathrm{MeV}$. The main contribution to the nucleon-nucleon interaction arises from the induced interaction [Fig. 2(d)], that associated with the bare nucleon-nucleon potential [cf. Fig. 2(a)] being quite small $(\approx 100 \mathrm{keV})$. This result is associated with the fact that, in ${ }^{12} \mathrm{Be}$, the single-particle states allowed to the valence neutrons to correlate are essentially the $s_{1 / 2}, p_{1 / 2}$, and $d_{5 / 2}$ orbits. Consequently the two neutrons are not able, in this low angular momentum phase space, to profit from the pairing contribution of the bare interaction which is connected with the high components of the associated multipole expansion.

The squared amplitudes of the ${ }^{12} \mathrm{Be}$ ground state wave function are shown in Table I [23]. The $d_{5 / 2}^{2}(0)$ amplitude predicted for the ${ }^{12} \mathrm{Be}$ ground state (cf. also Ref. [7]) is much larger than that calculated in the case of ${ }^{11} \mathrm{Li} \mathrm{[25].} \mathrm{This} \mathrm{is} \mathrm{due}$ to the fact that the $d_{5 / 2}$ orbital in ${ }^{10} \mathrm{Li}$ is much less confined than in ${ }^{11} \mathrm{Be}$, thus displaying much smaller overlaps with the $1 s_{1 / 2}$ and $0 p_{1 / 2}$ orbitals. Furthermore to the fact that in ${ }^{11} \mathrm{Li}$ the dipole mode is much softer than in ${ }^{12} \mathrm{Be}$ [30]. Using this wave function and that obtained for the ground state and the first excited state of ${ }^{11} \mathrm{Be}$, we have calculated the spectroscopic factors associated to the knockout of a single nucleon. In particular, a nucleon with quantum number $1 / 2^{-}$, $S\left[1 / 2^{-}\right]=\left|\left\langle{ }^{11} \mathrm{Be}\left|\widetilde{a}_{1 p_{1 / 2}}\right|{ }^{12} \mathrm{Be}\right\rangle\right|^{2}=\left|T_{1 / 2}\right|^{2}$, where $\widetilde{a}_{1 p_{1 / 2}}$ denotes the annihilation operator of the correlated $1 p_{1 / 2}$ singleparticle state in ${ }^{11} \mathrm{Be}$. In this calculation, use is made of the eigenvectors of the effective matrix associated with the lowest excited state of ${ }^{11} \mathrm{Be}$ and with the ground state of ${ }^{12} \mathrm{Be}$. We will indicate the former with $\widetilde{\xi}_{p}$ (the index $p$ indicating the different $p_{1 / 2}$ single-particle states of the basis) and the latter with $\widetilde{\xi}_{i i^{\prime}}$ (the index $i i^{\prime}$ indicating that the corresponding basis state contains a pair of particles moving in the levels $\left.i, i^{\prime}\right)$. In particular, one writes $\widetilde{a}_{1 p_{1 / 2}}=\sum_{n p_{1 / 2}} \widetilde{\xi}_{n p_{1 / 2}} a_{n p_{1 / 2}}$. The components $\widetilde{\xi}_{p}$ and $\widetilde{\xi}_{i i^{\prime}}$ are related to the so-called physical amplitudes, $\xi_{p}$ and $\xi_{i i}$, through the relations (cf., e.g., Ref. [12]) $\xi_{p}=\widetilde{\xi}_{p} / \sqrt{N\left({ }^{11} \mathrm{Be}\right)}$ and $\xi_{i i^{\prime}}=\widetilde{\xi}_{i i^{\prime}} / \sqrt{N\left({ }^{12} \mathrm{Be}\right)}$, where $N\left({ }^{11} \mathrm{Be}\right)$ and $N\left({ }^{12} \mathrm{Be}\right)$ are the normalization factors for ${ }^{11} \mathrm{Be}$ and ${ }^{12} \mathrm{Be}$, respectively. By using the physical amplitudes $\xi_{p}$ and $\xi_{i i^{\prime}}$ one obtains the value of $T_{1 / 2}$, 


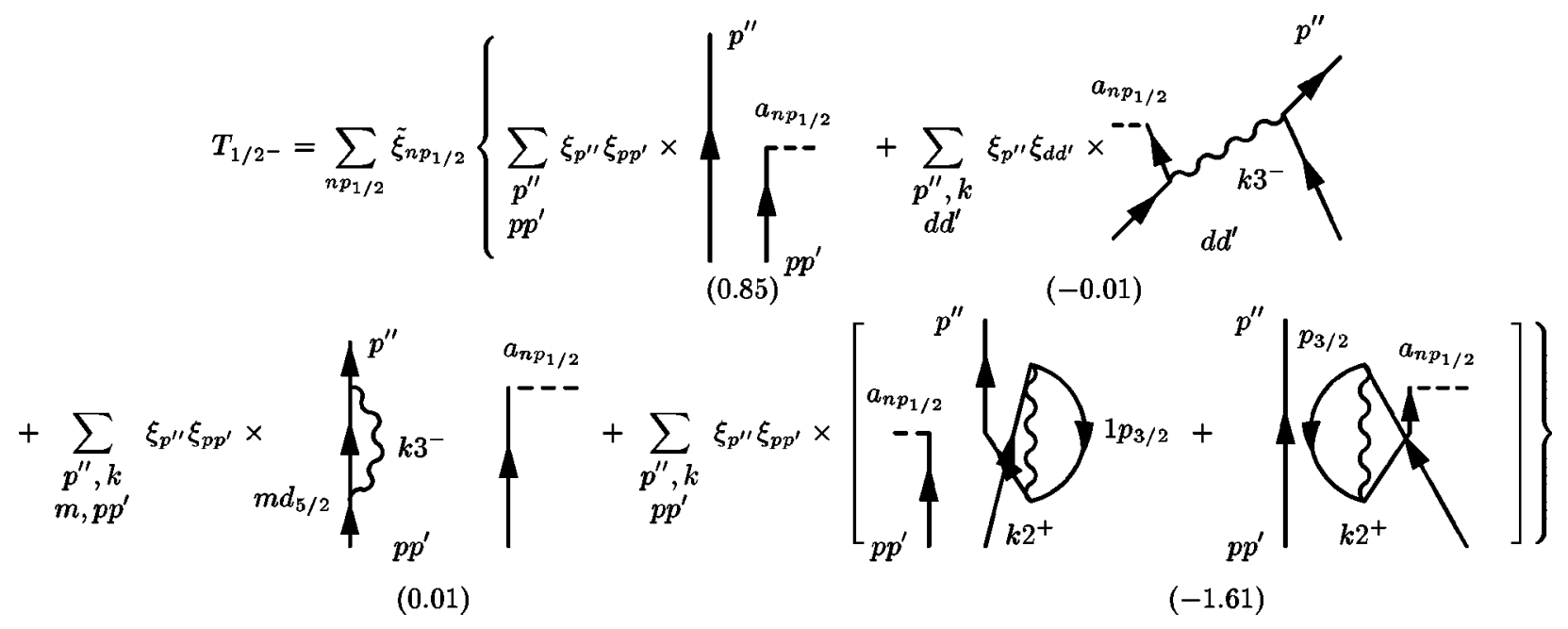

Summing up the different contributions (numbers in brackets), one obtains the spectroscopic factor $\left|T_{1 / 2^{-}}\right|^{2}$ =0.57. Similarly, one obtains $\left|T_{1 / 2^{+}}\right|^{2}=0.31$ (cf. Table I). Also the spectroscopic factors associated with ${ }^{11} \mathrm{Be}$ (Table I) were calculated following the same scheme.

Diagonalizing the matrix shown in Fig. 2, we also obtain the energies of the excited $0^{+}$states in ${ }^{12} \mathrm{Be}$. In particular, we obtain that the energy of the first excited state is $2.04 \mathrm{MeV}$, to be compared with the experimental value of $2.24 \mathrm{MeV}$ [31].
We conclude that the main nuclear structure properties of both ${ }^{11} \mathrm{Be}$ and ${ }^{12} \mathrm{Be}$ may be understood in terms of the selfenergy and induced interaction processes associated with the dynamic polarization of the nuclear surface. The similarity of NFT results with those of large shell model calculations reported in Ref. [7] for ${ }^{12} \mathrm{Be}$ and in Ref. [32] for ${ }^{11} \mathrm{Be}$, indicates that a proper treatment of single particle and of collective degrees of freedom and of their interweaving provides an essentially complete description of the nuclear structure of these nuclei as was already found in the case of nuclei lying along the stability valley.
[1] L. N. Cooper, Phys. Rev. 104, 1189 (1956).

[2] R. Kubo, J. Phys. Soc. Jpn. 17, 975 (1962).

[3] A. Bohr and B. R. Mottelson, Nuclear Structure (Benjamin, New York, 1969), Vol. I.

[4] A. Bohr and B. R. Mottelson, Nuclear Structure (Benjamin, New York, 1975), Vol. II.

[5] G. F. Bertsch and R. A. Broglia, Oscillations in Finite Quantal Systems (Cambridge University Press, Cambridge, 1991).

[6] D. J. Millener et al., Phys. Rev. C 28, 497 (1983).

[7] A. Navin et al., Phys. Rev. Lett. 85, 266 (2000).

[8] C. Mahaux et al., Phys. Rep. 120, 1 (1985).

[9] J. R. Schrieffer, Theory of Superconductivity (Benjamin, New York, 1964).

[10] H. Iwasaki et al., Phys. Lett. B 481, 7 (2000).

[11] G. Audi and A. H. Wapstra, Nucl. Phys. A595, 409 (1995).

[12] P. F. Bortignon et al., Phys. Rep., Phys. Lett. 30C, 305 (1977).

[13] R. B. Wiringa et al., Phys. Rev. C 29, 1207 (1984).

[14] D. J. Rowe, Nuclear Collective Motion (Methuen, London, 1970), p. 47.

[15] G. G. Dussel and R. Liotta, Phys. Lett. 37B, 477 (1971).

[16] J. S. Al-Khalili and J. A. Tostevin, Phys. Rev. Lett. 76, 3903 (1996).

[17] T. Aumann et al., Phys. Rev. Lett. 84, 35 (2000).
[18] G.-B. Liu and H. T. Fortune, Phys. Rev. C 42, 167 (1990).

[19] J. S. Winfield et al., Nucl. Phys. A683, 48 (2001).

[20] D. L. Auton, Nucl. Phys. A157, 305 (1970).

[21] B. Zwieglinski et al., Nucl. Phys. A315, 124 (1979).

[22] N. K. Timofeyuk et al., Phys. Rev. C C59, 1545 (1999).

[23] We observe that the state lying at $1.28 \mathrm{MeV}$ measured in ${ }^{11} \mathrm{Be}$ $[18,21]$ has been only tentatively assigned as a $d_{5 / 2}$ state. In our calculation, the $d_{5 / 2}$ state, after renormalization, lies very close to the edge of the continuum (cf. Table I). Changing the depth of the Saxon-Woods potential for the $d_{5 / 2}$ states, and setting the unperturbed energy of the resonance at $4.1 \mathrm{MeV}$, we could put the dressed resonance at $1.2 \mathrm{MeV}$. In this case the values of $S\left[1 / 2^{+}\right], S\left[1 / 2^{-}\right]$, and $S\left[5 / 2^{+}\right]$were $0.90,0.96$, and 0.73 , respectively. The ${ }^{12} \mathrm{Be}$ ground state wave function becomes $\left(s^{2}, p^{2}, d^{2}\right)=80 \%, 5 \%, 15 \%$.

[24] H. Sagawa et al., Phys. Lett. B 309, 1 (1993).

[25] F. Barranco et al., Eur. Phys. J. A 11, 385 (2001).

[26] N. Vinh Mau, Nucl. Phys. A592, 33 (1995).

[27] F. M. Nunes et al., Nucl. Phys. A596, 171 (1996).

[28] F. M. Nunes et al., Nucl. Phys. A609, 43 (1996).

[29] H. Esbensen et al., Phys. Rev. C 56, 3054 (1997).

[30] H. Iwasaki et al., Phys. Lett. B 491, 8 (2000).

[31] S. Shimoura et al., Phys. Lett. B 560, 31 (2003).

[32] T. Otsuka et al., Phys. Rev. Lett. 70, 1385 (1993). 